\title{
Evolution of TMD parton distributions up to NNLO approximation
}

\section{Roghayeh Taghavi}

Physics department, Yazd university, Yazd, Iran

E-mail: r.taghaviestu.vazd.ac.in

\section{Abolfazl Mirjalili* \\ Physics department, Yazd university, Yazd, Iran \\ E-mail: a.mirjalilievazd.ac.iv \\ Mohammad Mehdi Yazdanpanah \\ Faculty of Physics, Shahid Bahonar university of Kerman, Kerman, Iran \\ E-mail: myazdanduk.ac.in}

The transverse momentum dependent (TMD) parton distribution functions will provide us new insights into the substructure of nucleon beyond the one-dimensional (longitudinal) picture. Evidence for TMDs are accessible in processes such as semi-inclusive DIS (SIDIS) or dilepton produced in the Drell-Yan process. Here we are investigating the evolution of the unpolarized TMDs up to the NNLO approximation. The calculations are based on Collins-Sopper-Sterman formalism. The unknown parameters of non-perturbative part is being extracted via the fitting to the BNLY group experimental data. Comparison the evolved TMDs with each other at the NLO and NNLO approximations indicates that the contribution of NNLO approximation will be dominated at the high energy scale. This is expecting since at higher energy scale the gluon radiation is increasing and consequently the effect of parton transverse momentum would be outstanding. By accessing the TMD PDFs and also the numerical values of parameters in non-perturbative part of TMDs and in order to check the validity of calculations, it is possible to calculate the cross section in the Drell Yan processes which indicates a good agrement with the available experimental data.

The European Physical Society Conference on High Energy Physics

22-29 July 2015

Vienna, Austria

${ }^{*}$ Speaker. 


\section{Introduction}

If we consider only the longitudinal degrees of freedom of the partons, our PDF is called collineari. If, instead, we consider in addition the transverse component of the parton motion, we speak about Transverse Momentum Dependent PDFs (TMD PDFs or simply TMDs). One theoretical framework designed to account for transverse momentum effects is the resummation formalism developed by Collins-Sopper-Sterman (CSS)[U, [1]. This formalism is suitable for some processes that are sensitive to transverse momentum of parton like Drell-Yan (DY) process, semi inclusive deep inelastic scattering (SIDIS), and back-to-back hadron production in electron-positron annihilation. The TMD PDFs, like the ordinary collinear or integrated parton distribution functions (PDFs), are quantities which can be evolved between two different scales, so that all the non-perturbative QCD information encoded in a TMD PDF at one scale can be directly used at a different one by means of the appropriate evaluator. In fact, in practical point of view the evolution of TMD PDF is very important. Here, the evolution of TMD PDF in next-to-next-to-leading order is considering, based on the CSS formalism. On the other hand, the DY data is a good case to test the CSS formalism. So, in this paper, the cross section data of DY experiment was considered.

\section{TMD evolution}

In the Collins-Soper-Sterman (CSS) formalism, The evolution of a quark-TMDPDF is given by [3], 团]:

$$
\begin{array}{r}
\overbrace{\tilde{F}_{f / P}\left(x, b_{T} ; \mu, \zeta_{F}\right)=\overbrace{\sum_{j} \int_{x}^{1} \frac{d \hat{x}}{\hat{x}} \tilde{C}_{f / j}\left(x / \hat{x}, b_{*} ; \mu_{b}{ }^{2}, \mu_{b}, g\left(\mu_{b}\right)\right) f_{j / P}\left(\hat{x}, \mu_{b}\right)}^{A}} \\
\times \overbrace{\exp \left\{\ln \frac{\sqrt{\zeta_{F}}}{\mu_{b}} \tilde{K}\left(b_{*} ; \mu_{b}\right)+\int_{\mu_{b}}^{\mu} \frac{d \mu^{\prime}}{\mu^{\prime}}\left[\gamma_{F}\left(g\left(\mu^{\prime}\right) ; 1\right)-\ln \frac{\sqrt{\zeta_{F}}}{\mu^{\prime}} \gamma_{K}\left(g\left(\mu^{\prime}\right)\right)\right]\right\}}^{C} \\
\times \overbrace{\exp \left\{g_{j / P}\left(x, b_{T}\right)+g_{K}\left(b_{T}\right) \ln \frac{\sqrt{\zeta_{F}}}{\sqrt{\zeta_{F, 0}}}\right\}}^{B}
\end{array}
$$

In this equation the $\tilde{C}_{f / j}\left(x / \hat{x}, b_{*} ; \mu_{b}{ }^{2}, \mu_{b}, g\left(\mu_{b}\right)\right), \tilde{K}\left(b_{*} ; \mu_{b}\right), \gamma_{K}\left(g\left(\mu^{\prime}\right)\right)$ and $\gamma_{F}\left(g\left(\mu^{\prime}\right) ; 1\right)$ functions are all perturbatively calculable for all $b_{T}$ and on the first line of this equation $f_{j / P}\left(\hat{x}, \mu_{b}\right)$ is the ordinary PDF from. The functions $g_{j / P}\left(x, b_{T}\right)$ and $g_{K}\left(b_{T}\right)$ are related to the non-perturbative $b_{T}$ behavior of $\tilde{F}_{f / P}\left(x, b_{T} ; \mu, \zeta_{F}\right)$ and $K\left(b ; \mu_{b}\right)$ respectively. The property of these functions is that they are scale-independent and universal where $\gamma_{F}, \gamma_{K}$ are the anomalous dimensions of the $\tilde{F}_{f / P}\left(x, b_{T} ; \mu, \zeta_{F}\right)$ and $K\left(b ; \mu_{b}\right)$ functions. In fact, in Eq. (2.1) the A-factor matches the TMD PDF to a collinear treatment in the small $b_{T} \ll \frac{1}{\Lambda_{Q C D}}$ limit. This factor involves a hard part which in this case is the coefficient function $\tilde{C}_{f / j}$ and a collinear factor which is just the standard integrated PDF. The B-factor is an exponential of quantities that can all be calculated perturbatively. The last factor the $\mathrm{C}$-factor implements the matching between the small and large $b_{T}$-dependence. The function $g_{j / P}\left(x, b_{T}\right)$ parameterizes the non-perturbative large- $b_{T}$ behavior that is intrinsic to the 
proton while $g_{K}\left(b_{T}\right)$ parameterizes the non-perturbative large- $b_{T}$ behavior of $\tilde{K}\left(b_{*} ; \mu_{b}\right)$. The function $g_{j / P}\left(x, b_{T}\right)$ is universal but it depends on the external hadron. The function $g_{K}\left(b_{T}\right)$ is universal and also independent of the species of external hadrons. For doing calculations, as is expecting, we choose $\sqrt{\zeta_{F}}=Q$ and $\sqrt{\zeta_{F, 0}}=Q_{0}$. In this research the TMD PDF of up quark at the NNLO approximation for the small, medium and large values of $Q=\sqrt{2.4}, 5$, and $91.19 \mathrm{GeV}$ with $\mathrm{x}=$ 0.09 are obtained. We have made the plot run over a range from $k_{T}=0$ to $6 \mathrm{GeV}$ typical for studies of TMD-functions. To do the calculations we need to the one-loop values for $\tilde{K}\left(b_{*} ; \mu_{b}\right), \gamma_{F}$ and $\gamma_{K}$ functions which are [四]:

$$
\begin{gathered}
\tilde{K}\left(\mu, b_{T}\right)=-\frac{\alpha_{s} C_{F} L_{\perp}}{\pi}, \\
\gamma_{F}(\mu)=\frac{\alpha_{s} C_{F}}{\pi}\left(\frac{3}{2}-\ln \left(\frac{\zeta_{F}}{\mu^{2}}\right)\right), \\
\gamma_{K}(\mu)=2 \frac{\alpha_{s} C_{F}}{\pi}
\end{gathered}
$$

where

$$
L_{\perp}(\mu, b t)=\log \left(\frac{\mu^{2} b_{t}^{2}}{4 e^{-2 \gamma_{E}}}\right) .
$$

By considering these terms at the NLO approximation the TMD PDF for up quark in three different scales are calculated. Then by adding the NNLO terms to the equations (2.2), (2.3) and (2.4) we have [1]]:

$$
\begin{gathered}
\tilde{K}\left(\mu, b_{T}\right)=-\frac{\alpha_{s} C_{F} L_{\perp}}{\pi}+\left(\frac{\alpha_{s}}{\pi}\right)^{2}\left(\frac{1}{32} \Gamma_{0} \beta_{0} L_{\perp}\left(\mu, b_{T}\right)^{2}-\frac{1}{8}\left(\frac{\Gamma_{1}}{2} L_{\perp}\left(\mu, b_{T}\right)\right)-\frac{1}{8} d_{20}\right), \\
\gamma_{F}\left(\mu ; \zeta_{F} / \mu^{2}\right)=\alpha_{s} \frac{C_{F}}{\pi}\left(\frac{3}{2}-\ln \left(\frac{\zeta_{F}}{\mu^{2}}\right)\right)+\left(\frac{\alpha_{s}(\mu)}{\pi}\right)^{2}\left(-4 C_{F}\left(\left(\frac{67}{9}-\frac{\pi^{2}}{3}\right) C_{A}-\frac{20}{9} T_{F} N_{f}\right)\left(\log \left[\frac{\zeta}{\mu^{2}}\right]\right)-\right. \\
\left.C_{F}^{2}\left(-3+4 \pi^{2}-48 \varsigma_{3}\right)-C_{F} C_{A}\left(-\frac{961}{27}-\frac{11 \pi^{2}}{3}+52 \varsigma_{3}\right)-C_{F} T_{F} N_{f}\left(\frac{260}{27}+\frac{4 \pi^{2}}{3}\right)\right), \\
\gamma_{K}(\mu)=2 \frac{\alpha_{s} C_{F}}{\pi}+2\left(\frac{\alpha_{s}}{\pi}\right)^{2}\left(C_{F} C_{A}\left(\frac{67}{36}-\frac{\pi^{2}}{12}\right)-\frac{5}{18} C_{F} N_{f}\right),
\end{gathered}
$$

where:

$$
\begin{gathered}
\Gamma_{0}=4 C_{F}, \\
\beta_{0}=\frac{11}{3} C_{A}-\frac{4}{3} T_{F} N_{f},
\end{gathered}
$$




$$
\begin{gathered}
\Gamma_{1}=4 C_{F}\left(\left(\frac{67}{9}-\frac{\pi^{2}}{3}\right) C_{A}-\frac{20}{9} T_{F} N_{f}\right), \\
d_{20}=C_{F} C_{A}\left(\frac{404}{27}-14 \varsigma_{3}\right)-\left(\frac{112}{27}\right) C_{F} T_{F} N_{f} .
\end{gathered}
$$

Finally, by numerical calculations and employing Eqs.(2.6), (2.7) and (2.8) in Eq. (2.1), the TMD PDF of up quark at the NNLO approximation for the small, medium and large values of $Q=\sqrt{2.4}$ , 5, and $91.19 \mathrm{GeV}$ and with $\mathrm{x}=0.09$ in $Q_{0}=1.6 \mathrm{GeV}$ can be obtained. We have made the plots run over a range from $k_{T}=0$ to $6 \mathrm{GeV}$ and depicted them in Fig. 1 .

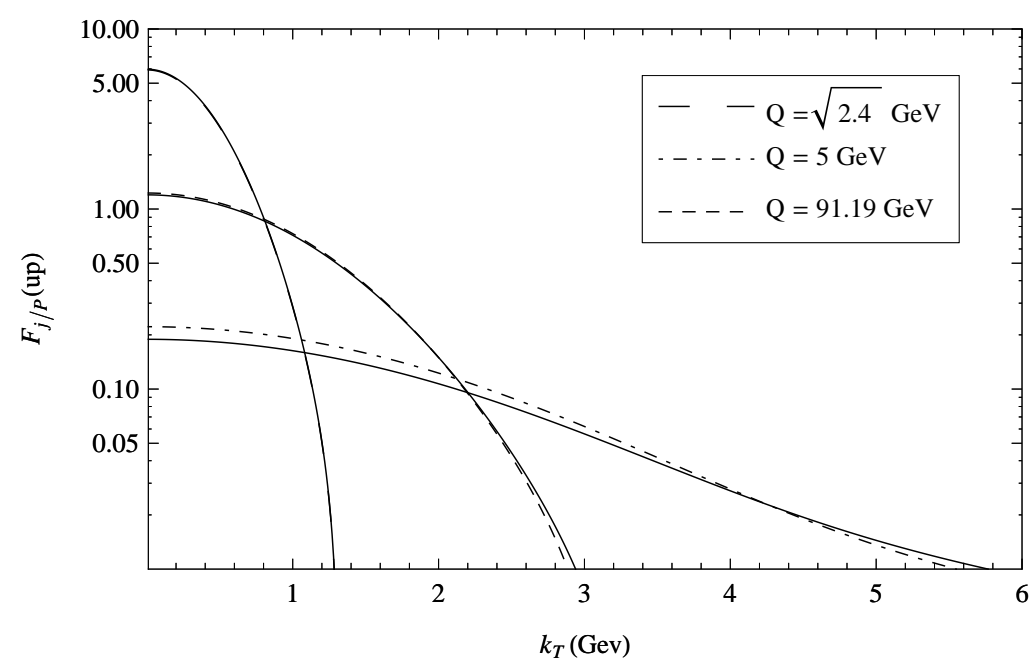

Figure 1: The up quark TMD PDF for $Q=\sqrt{2.4}, 5.0$ and $91.19 \mathrm{GeV}$ and $\mathrm{x}=0.09$. The plots indicate the results at the NLO (dashed curves) and NNLO (solid curves) approximations with $b_{\max }=0.5 \mathrm{GeV}^{-1}$.

\section{Drell-Yan cross section}

After obtaining the TMD PDF of up quark at the NNLO approximation we consider the DrellYan production of lepton pairs. In this type of processes the differential cross section can be expressed as [目]:

$$
\frac{d \sigma}{d Q^{2} d Q_{T}^{2} d y}=\frac{1}{(2 \pi)^{2}} \delta\left(Q^{2}-M_{V}^{2}\right) \int d^{2} b e^{i \vec{Q}_{T} \cdot \vec{b}_{T}} \tilde{W}_{j \bar{k}}\left(b, Q, x_{1}, x_{2}\right)+Y\left(Q, Q_{T}, x_{1}, x_{2}\right),
$$

where $Q, Q_{T}, y, x_{1}, x_{2}$ are the invariant mass, transverse momentum, rapidity of vector boson $\mathrm{V}$, $x_{1}=e^{y} Q / s, x_{2}=e^{-y} Q / s$ respectively and $\mathrm{s}$ is the overall center-of-mass energy. The $\mathrm{W}$ term in Eq.(3.1) is defined as [目]:

$$
\widetilde{W}_{j \bar{k}}\left(b, Q, x_{1}, x_{2}\right)=e^{-S\left(Q, b, C_{1}, C_{2}\right)} \sum_{j, \bar{k}} \frac{\sigma_{0}}{S} \widetilde{F}_{j / 1}\left(x_{1}, b_{T}, \zeta_{1}, \mu\right) \widetilde{F}_{\bar{k} / 2}\left(x_{2}, b_{T}, \zeta_{2}, \mu\right)+(j \leftrightarrow \bar{k}),
$$


where 1,2 refres to the parton number 1 and parton number 2 . The Sudakov factor $\mathrm{S}$ is defined as [焑]:

$$
S\left(Q, b, C_{1}, C_{2}\right)=\int_{C_{1}{ }^{2} / b^{2}}^{C_{2}{ }^{2} Q^{2}} \frac{d \bar{\mu}^{2}}{\bar{\mu}}\left[\mathrm{A}\left(\alpha_{s}(\bar{\mu}), C_{1}\right) \ln \left(\frac{C_{2}{ }^{2} Q^{2}}{\bar{\mu}^{2}}\right)+B\left(\alpha_{s}(\bar{\mu}), C_{1}, C_{2}\right)\right] .
$$

We have analyzed the Drell-Yan process with Brock-Landry-Nadolsky-Yuan (BLNY) parametrization in the non-perturbative part of the cross section fitting with the form:

$$
\exp \left\{\left[-g_{1}-g_{2} \ln \left(\frac{Q}{2 Q_{0}}\right)-g_{1} g_{3} \ln \left(100 x_{1} x_{2}\right)\right] b^{2}\right\},
$$

where $g_{1}, g_{2}, g_{3}$ are the unknown parameters that can be determined by fitting to the available experimental data. Using the $\mathrm{E} 288$ experimental data (for $\mathrm{Q}=5$ to $9 \mathrm{GeV}$ ) the numerical values of these parameters can be obtained via the fitting. This is done by the $b$ star method $[\mathbb{}]$ and we choose $b_{\max }=0.5 \mathrm{GeV}^{-1}=0.1 \mathrm{fm}$ in all fits. We can then calculate the theoretical cross section. In order to compare this theoretical result with experimental data we need to multiply the data by suitable normalization factor. After performing fitting procedure the values of non perturbative parameters in $5<\mathrm{Q}<6$ mass bin are obtained as it follows:

$$
g_{1}=0.49, g_{2}=0.56, g_{3}=-0.56
$$

Comparison of theoretical calculation of cross section to E288 data for the $p+C u \rightarrow \mu^{+} \mu^{-}+X$ process at $\sqrt{s}=27.4 \mathrm{GeV}$ and $\mathrm{y}=0.03$ have been shown in Fig.2.

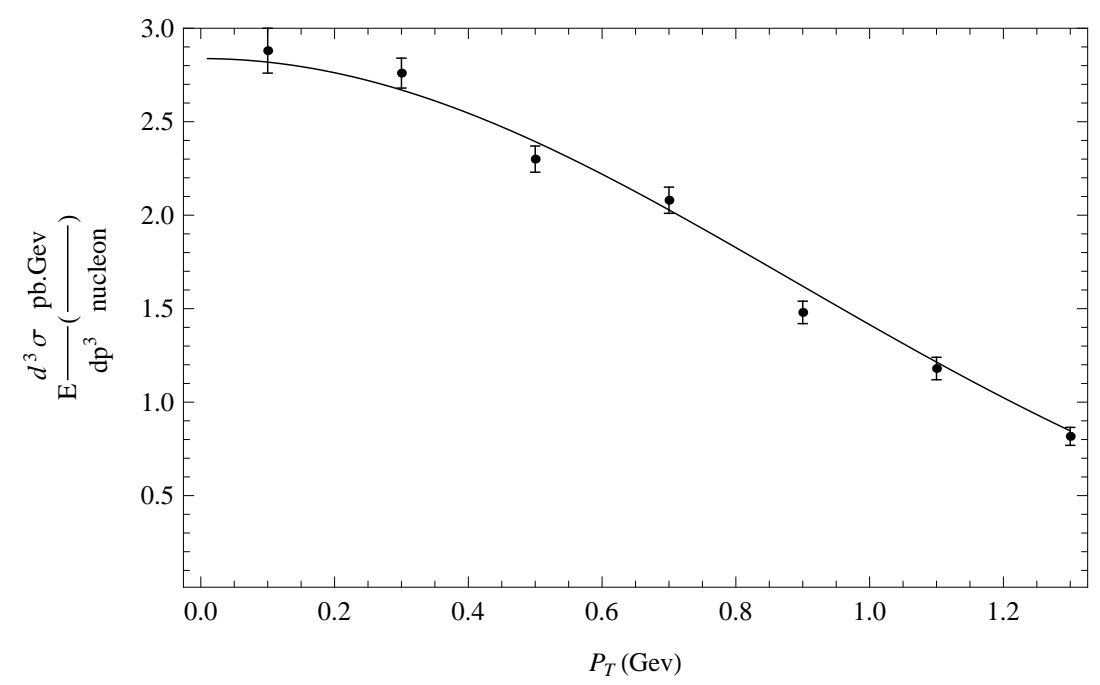

Figure 2: Comparison of theoretical calculation of cross section in $5<\mathrm{Q}<6$ mass bin to E288 data [8] for the $p+C u \rightarrow \mu^{+} \mu^{-}+X$ process at $\sqrt{s}=27.4 \mathrm{GeV}$

Then one can conclude that the CSS is a valid factorization theorem in which theoretical calculation of cross section based on this formalism have a good match to experimental data. 


\section{Conclusion}

The TMD formalism is a powerful tool to analyze perturbative and non-perturbative effects in hadronic spectra. In this work, we have considered the evolution of transverse momentum dependent parton distribution function for up quark in CSS formalism. In particular, we have used the TMD evolution kernel at the NNLO approximation which, to our knowledge, has not been done before. One important outcome of this work is that the evolution with energy scale $\mathrm{Q}$ has more effect at high scales. In fact this effect is not considerable at low energy scale. Because in high energy scales the emission of gluons is more dominant and by including the effect of multiple gluon emission the transverse momentum of parton will be more effective.

Another outcome of this work is that the CSS formalism can describe properly the E288 experimental data in the Drell-Yan process. The good agrement between theoretical result and the available experimental data for the production cross section of vector boson in collision of two hadrons, indicates the validity of our calculations for the TMD PDFs at the NNLO approximation.

\section{Acknowledgment}

Authors are indebted to the research council of Yazd university to provide the required facilities to attend in EPS-HEP2015 conference. We are also thankful to the organizers for their efforts to provide a very attractive environment during the conference.

\section{References}

[1] ] J. C. Collns, D. Sterman, Nucl. Phys. B 250 (1985) 199.

[2] L. N. Lipatov, Sov. J. Nucl. Phys. 20 (1975) 95.

[3] J. C. Collins, Foundations of Perturbative QCD ,Cambridge University Press, Cambridge, (2011).

[4] Mert Aybat, and Ted C. Rogers, Phys.Rev. D 83 (2011) 114042.

[5] T. Gehrmann, T. Lubbertb, L. L. Yang, JHEP 1406 (2014) 155.

[6] F. Landry, R. Brock, P. M. Nadolsky, C. P. Yuan, Phys.Rev. D 67 (2003) 073016.

[7] Miguel G. Echevaria, Ahmad Idilbi, Andreas Schafer and Ignazio Scimemi, Eur.Phys.J. C 73 (2013) 12.

[8] A.S. Ito (SUNY, Stony Brook), Phys.ReV.D 23 (1981) 604. 